\title{
AIRBORNE LIDAR OBSERVATIONS OF WATER VAPOR TRANSPORT
}

\author{
Christoph Kiemle, Andreas Schäfler, Martin Wirth, Andreas Fix and Stephan Rahm
}

\author{
Lidar Group, Institut für Physik der Atmosphäre, Deutsches Zentrum für Luft- und Raumfahrt (DLR), \\ 82234 Oberpfaffenhofen, Germany
}

\begin{abstract}
Water vapor, a minor constituent of the earth's atmosphere, plays a major role in the radiation budget and the water cycle with important implications for weather and climate. Due to the heterogeneous distribution of its sources, evaporation, and sinks, condensation and precipitation, and due to the complexity of atmospheric motion and mixing, its distribution is highly variable. Despite the relevance of this trace gas, its variability and instrumental shortcomings impede accurate measurements of its concentration. Advanced airborne lidar instruments aid to better observe water vapor and its transport in the atmosphere, in view of an improved understanding of the related key implications. The combination of a water vapor differential absorption lidar and a heterodyne detection Doppler wind lidar on an aircraft allows to measure vertical profiles of the latent heat flux in a convective boundary layer and to portray the smallto meso-scale humidity transport and variability beneath the aircraft with high accuracy and spatial resolution, in the frame of dedicated meteorological process studies.
\end{abstract}

Index Terms - Airborne lidar, water vapor transport, latent heat flux, differential absorption, boundary layer

\section{INTRODUCTION}

Water vapor is routinely measured at the surface, with the global radiosonde network and with satellite sensors. The radiosondes' accuracy is sufficient in the lower and middle troposphere, but the representativeness of the onedimensional vertical profiles is poor. Furthermore, few data are collected over the oceans, at high latitudes, and in the southern hemisphere. In these regions, and in the upper troposphere, satellite spectrometers partly fill the observational gap, but with coarse spatial resolution. Better accuracy and resolution is obtained by active, optical lidar remote sensing. Since 1987 the DLR atmospheric physics institute's lidar group develops and operates airborne water vapor lidars. Participation in numerous field experiments helped characterize the distribution of water vapor in the frame of case studies dedicated to specific physical and meteorological processes. A recent instrumental highlight is

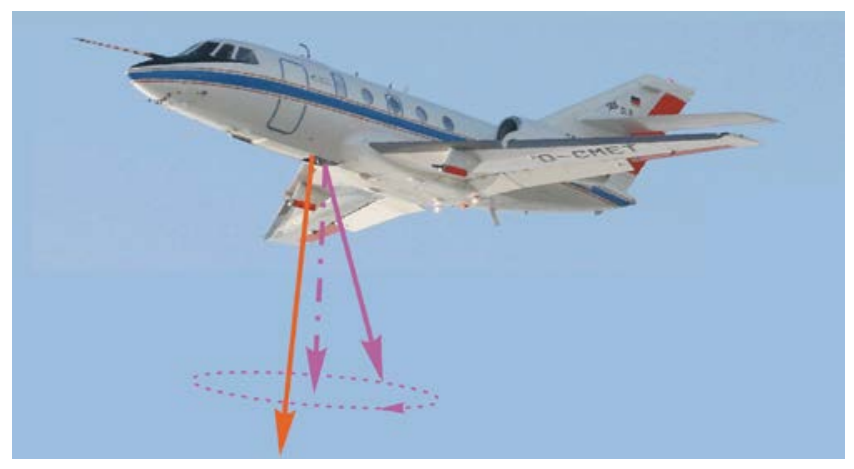

Figure 1: DLR Falcon research aircraft with idealized wind (pink) and water vapor (orange) lidar beams. The wind lidar can be operated either in conical scanning mode for estimates of the three-dimensional wind vector, or in nadir viewing mode for vertical wind velocity measurements.

\begin{tabular}{lll} 
& DIAL & Wind Lidar \\
\hline Transmitter type & OPO & Tm:LuAG \\
Wavelength (nm) & 935 & 2022 \\
Pulse energy (mJ) & 40 & 1.5 \\
PRF (Hz) & 200 & 500 \\
Average power (W) & 8 & 0.75 \\
Detection principle & direct & heterodyne \\
Detector type & APD & PIN diode \\
Telescope diam. (cm) & 48 & 10 \\
Precision & $0.4 \mathrm{~g} / \mathrm{kg}$ & $0.1 \mathrm{~m} / \mathrm{s}$ \\
\hline
\end{tabular}

Table 1: Main characteristics of airborne water vapor differential absorption lidar (DIAL) and Doppler wind lidar.

the co-deployment of a newly developed water vapor and a wind lidar onboard the DLR Falcon research aircraft (Figure 1; Table 1).

The difference between a laser emission wavelength tuned to the center of a $\mathrm{H} 2 \mathrm{O}$ absorption line and a non-absorbed off-line reference is proportional to the $\mathrm{H} 2 \mathrm{O}$ molecule density in air. The DIAL has two transmitters, each based on an injection-seeded optical parametric oscillator (OPO) pumped by the second harmonic of a Q-switched, diode-pumped single-mode Nd:YAG laser at a pulse 




Figure 2: Airborne water vapor lidar measurements over complex terrain in Southwest Germany, on 30 July 2007 at noon. Blue represents dry air above, yellow and red show humid air within the convective boundary layer (CBL). The length $\times$ height of the data curtains is $150 \mathrm{~km} \times 4 \mathrm{~km}$. The topographic heights range from $150 \mathrm{~m}$ to $1500 \mathrm{~m}$. Interruptions are due to scattered fair-weather clouds at the CBL top.

repetition frequency (PRF) of $100 \mathrm{~Hz}$ [1]. The OPO is optimized to operate in the spectral region between 920 $950 \mathrm{~nm}$ at average output pulse energy of $40 \mathrm{~mJ}$. Each transmitter outputs two spectrally narrow pulses, yielding in total four pulses (each $50 \mathrm{~Hz}, 40 \mathrm{~mJ}$ ) at different wavelengths for different water vapor absorption lines. The wind lidar is operated either in conical-scanning mode with $20^{\circ}$ off-nadir angle for 3 -d wind field profiles, or in nadirviewing mode for vertical wind speed profiles.

\section{VERTICAL WATER VAPOR TRANSPORT, LATENT HEAT FLUX}

Evaporation over land and especially over complex, mountainous terrain can be very heterogeneous on small scales that are both under-sampled by observations, and unresolved by numerical weather prediction (NWP) models. Over a land surface heated by solar irradiation, a convective boundary layer (CBL) develops, typically reaching heights of $1-2 \mathrm{~km}$ above ground in the afternoon. The buoyancy of warm air parcels transports the humidity vertically away from its surface sources. Cloud condensation at the boundary layer top, and subsequent cloud growth with occasionally heavy precipitation may occur. Observational and modeling deficits due to the heterogeneity of surface and atmospheric moisture are main reasons why numerical weather prediction (NWP) models fail to accurately forecast the precipitation in the warm season, even if evaporation and subsequent humidity transport are key elements of the hydrological cycle.

Consequently, a field experiment called "Convective and Orographically-induced Precipitation Study” (COPS) was conducted in July 2007 over the Black Forest low-mountains in south-western Germany, with the participation of the DLR Falcon aircraft. Figure 2 exemplarily shows two-dimensional vertical cross-sections of humidity beneath the aircraft along the flight track. This kind of data gives a decisive advantage over conventional one-dimensional "in-situ” measurements, particularly in this heterogeneous situation, where e.g. higher humidity is observed in the Rhine valley. One of the main goals of the DLR Falcon was to measure the vertical humidity transport within the CBL over the Rhine valley and the Black Forest on days when thunderstorms, mainly influenced by the orography and surface humidity, were forecasted [2]. From the remotely-sensed wind and water vapor fluctuations in the convective boundary layer, a representative area-averaged latent heat flux profile is obtained by the eddy-correlation technique. The lidars' horizontal and vertical resolution is $200 \mathrm{~m}$, sufficient to resolve the dominant contributions to the flux. The lidar-derived latent heat fluxes over the mountains vary between $100-500 \mathrm{~W} / \mathrm{m}^{2}$ but are roughly constant with height. The positive fluxes moisten the growing CBL by upward transport of humidity from surface evaporation due to previous days' rain. 


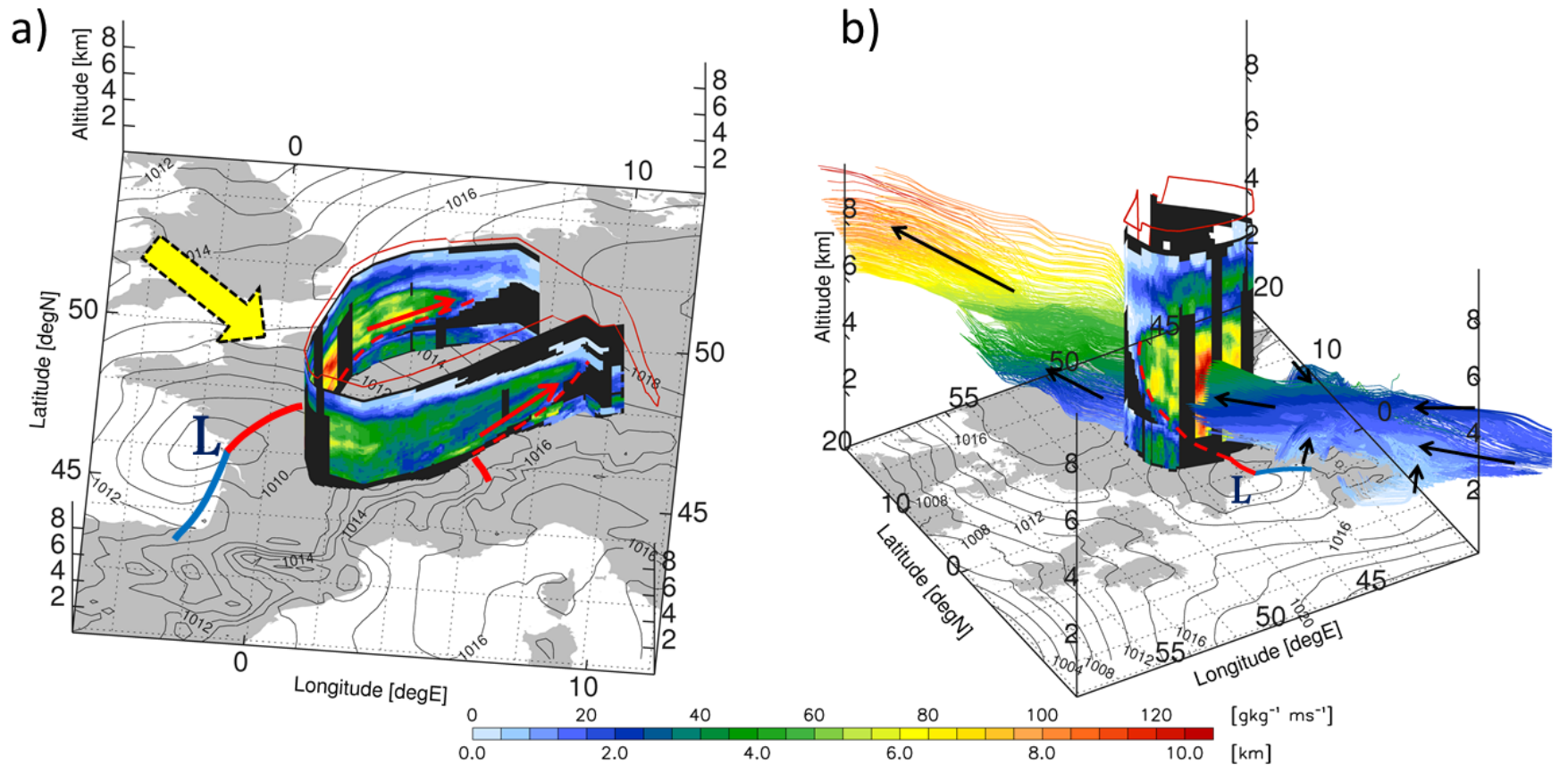

Figure 3: Airborne observations of water vapor transport on 1 August 2007 from combined water vapor and wind lidars. Perspective from the south (a) and from northwest (b), indicated by the yellow arrow in (a). The aircraft flew along the thin red line. The curtain-like 2-dimensional cross-section shows the transport of water vapor beneath the aircraft. Blue represents weak transport; yellow and red mean strong transport. Thin black contour lines on the map show the surface pressure [hPa]. The thick blue and red lines depict the location of the surface cold and warm front, respectively. The dashed red lines show the vertical extension of the warm front along the lidar curtain. In addition, (b) shows selected trajectories of air parcels with the maximum observed water vapor transport. Their colors indicate the altitude of the parcels for 2.5 days in advance and 1.5 days after the flight. Data gaps are black.

\section{HORIZONTAL WATER VAPOR TRANSPORT}

In the mid-latitudes between 30 and $60^{\circ} \mathrm{N}$, cyclones effectively remove energy differences between polar and subtropical areas as a result of differing solar radiation by transporting moist and warm air pole-ward and cold and dry air equator-ward. Typically, the polar and subtropical air masses are separated by a narrow band in the mid-latitudes possessing a sharp meridional temperature change. Along this frontal zone, low pressure systems evolve that transport air horizontally and vertically, generating cloud development and precipitation. Due to the minor depth of the layer where weather happens, the troposphere $(0-12 \mathrm{~km})$, most atmospheric transport processes can be considered quasi-horizontal. Advanced observation techniques like lidar are required to observe the water vapor transport in low pressure systems. Besides the understanding of the physical processes, the verification of model simulations by independent observations helps pinpointing insufficiencies in current NWP models.
Figure 3 shows an example from the COPS field experiment to highlight the value of high resolution lidar data to observe the highly-complex three-dimensional transport of moisture in the surrounding of a low pressure system. The curtain-like two-dimensional cross section represents a snapshot of the water vapor transport, determined by the product of wind velocity and water vapor concentration, and reveals an up to $6 \mathrm{~km}$ deep layer of enhanced water vapor transport in the warm sector of the cyclone [3]. Maximum values occur on the most western part of the flight facing the cold front, where increased wind speeds and water vapor concentrations coincide. Nicely, the strong horizontal gradients of the moisture transport at the warm front show a tilting with height, coming along with the northeastward ascending air masses (red dashed lines along the lidar cross section). The tilting is observed on both overpasses of the warm front. Red arrows denote the upgliding of the warm and moist air above the colder air to the north. In contrast, in the north-eastern part the horizontal transport in the boundary layer is weak. 
Figure 3b shows the same scenery from a northwestern perspective. The increased transport on the western side that extends upward along the tilted warm front is clearly visible. The figure also displays trajectories that describe the pathways of the observed air parcels in space and time. Shown are the pathways up to 2.5 days in advance and up to 1.5 days after the lidar observation. The trajectories were calculated using NWP wind data. The strong moisture transport clearly originates from the southwest. Air in the lower troposphere is humidified over the Atlantic and the Mediterranean by evaporation and subsequent vertical transport in the boundary layer. These air masses converge over southeastern Europe. The developing cyclone incorporates and transports the moisture northeastward towards the flight path. Many air parcels slightly ascend towards the lidar cross section and further up until they reach the upper troposphere ( 8 km) 1.5 days after being observed by the lidar. An in-depth discussion of this case study and a comparison with NWP data is found in [3].

The airborne lidar instruments are able to map the clear-air transport of water vapor into cyclones that is known to play a key role in the structure, intensity, and further evolution of cyclones. The latent heat release is expected to amplify cyclones, and also to affect the upper-level wind fields which control the weather development further downstream. This emphasizes the importance of a correct representation of moisture for the quality of weather forecasts. Further studies show that the lidar observations are useful for diagnosing NWP model errors which result from either incorrect horizontal or vertical transport of moisture or from insufficiently represented evaporation in moisture source regions.

\section{CONCLUSIONS AND OUTLOOK}

Accurate water vapor measurements are sparse and challenging because of the high variability of its sources in space and time, and because of the complexity of atmospheric motion and mixing. Advanced airborne water vapor observations are, as shown, valuable for addressing relevant issues of current research in atmospheric water vapor and transport. There is an increasing need for improved measurements, for example of the relative humidity in the upper troposphere in regions with ice supersaturation. This implies having simultaneous information on temperature from NWP data or auxiliary measurements. A combined water vapor and temperature lidar is a future challenging task, as temperature profiling by lidar is basically possible yet still subject to high detection noise, which necessitates long averaging periods and consequently means coarse spatial resolution.

Figure 4 shows the various and manifold interactions of water vapor with the atmosphere. Water vapor in the lower troposphere is particularly relevant for weather, while water vapor in the upper troposphere and the stratosphere is particularly relevant for climate, where, after vertical transport from the surface sources, it strongly influences Earth's radiation budget. Consequently, understanding the transport and distribution of atmospheric humidity and the processes that control it is of primary importance to both climate research and weather prediction. New instrument developments such as multi-wavelength lidar will, on board the new HALO research aircraft, advance the science, building on the knowledge gained in the above-mentioned experiments. The measurements will help to understand significant atmospheric processes and to evaluate state-of-the-art weather and climate models. In addition, future satellite missions to measure wind (ESA Atmospheric Dynamics Mission) or water vapor (ESA WALES: Water vapor Lidar Experiment in Space) will be supported and validated.

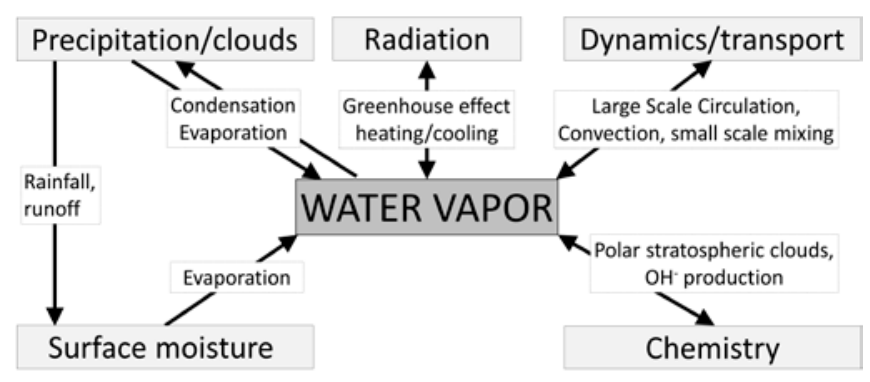

Figure 4: Interactions of water vapor with components of the climate system. The hydrological cycle is sketched on the left.

\section{REFERENCES}

[1] Wirth M., A. Fix, P. Mahnke, H. Schwarzer, F. Schrandt, G. Ehret, 2009: The airborne multi-wavelength water vapor differential absorption lidar WALES: system design and performance. Appl. Phys. B, 96, 201-213, DOI 10.1007/s00340009-3365-7.

[2] Kiemle C., M. Wirth, A. Fix, S. Rahm, U. Corsmeier, P. Di Girolamo, 2011: Latent heat flux measurements over complex terrain by airborne water vapour and wind lidars. Q. J. R. Meteorol. Soc. 137, 190 - 203.

[3] Schäfler, A., A. Dörnbrack, C. Kiemle, S. Rahm, M. Wirth, 2010: Tropospheric Water Vapor Transport as Determined from Airborne Lidar Measurements. J. Atmos. Ocean. Technol., 27, 2017-2030. 